\title{
THE NOVIKOV CONJECTURE
}

\author{
GUOLIANG YU \\ Dedicated to Sergei Novikov on the occasion of his 80th birthday
}

\begin{abstract}
We give a survey on recent development of the Novikov conjecture and its applications to topological rigidity and non-rigidity.
\end{abstract}

\section{INTRODUCTION}

A central problem in mathematics is the Novikov conjecture $[\mathrm{N}]$. Roughly speaking, the Novikov conjecture claims that compact smooth manifolds are rigid at an infinitesimal level. More precisely, the Novikov conjecture states that the higher signatures of compact oriented smooth manifolds are invariant under orientation-preserving homotopy equivalences. Recall that a compact manifold is called aspherical if its universal cover is contractible. In the case of aspherical manifolds, the Novikov conjecture is an infinitesimal version of the Borel conjecture, which states that all compact aspherical manifolds are topologically rigid, i.e. if another compact manifold $N$ is homotopy equivalent to the given compact aspherical manifold $M$, then $N$ is homeomorphic to $M$. A deep theorem of Novikov says that the rational Pontryagin classes are invariant under orientation-preserving homeomorphisms [N1]. The Novikov conjecture for compact aspherical manifolds follows from the Borel conjecture and Novikov's theorem since for aspherical manfolds, the information about higher signatures is equivalent to that of rational Pontryagin classes.

The Novikov conjecture has inspired a lot of beautiful mathematics. It motivated the development of Kasparov's $K K$-theory, Connes' cyclic cohomology theory, Gromov-ConnesMoscovici theory of almost flat bundles, Connes-Higson's E-theory, and quantitative operator $K$-theory. The Novikov conjecture has been proven for a large number of cases. The general philosophy is that the conjecture should be true if the fundamental group of the manifold arises from nature. The purpose of this article is to give a friendly survey on recent development of the Novikov conjecture and its applications to topological rigidity and non-rigidity.

There are two natural approaches to the Novikov conjecture: the analytic approach based on ideas from noncommutative geometry and the topological approach using $L$-theory. Up until this point, the analytic approach has been more successful partly due to the crucial fact that it is easier to do cutting and pasting in the world of noncommutative geometry. Cutting and pasting makes it possible to retrieve the $K$-theoretic information necessary to prove the

The author is partially supported by a grant from the U.S. National Science Foundation. 
Novikov conjecture. In noncommutative geometry, we are dealing with group $C^{*}$-algebras, while in topology, we are studying group rings. To illustrate this fundamental point, let us consider the case when the fundamental group is the group of integers. In this case, the group $C^{*}$-algebra is the algebra of continuous functions on the circle while the group ring is the ring of Laurent polynomials. We can apply cutting and paste to a continuous function to obtain another continuous functions using partitioning of unity, this procedure cannot be applied to Laurent polynomials.

This survey focuses on the analytic approach to the Novikov conjecture and as well as methods inspired by the analytic approach. The analytic approach uses operator $K$-theoretic techniques from noncommutative geometry [M, BC, C, K]. The Novikov conjecture follows from the rational strong Novikov conjecture, which states that the rational Kasparov-BaumConnes map is injective, here the Kasparov-Baum-Connes map is the assembly map from $K$-homology group of the classifying space for the fundamental group to the $K$-theory of group $C^{*}$-algebra associated to the fundamental group. Using this approach, the Novikov conjecture has been proven when the fundamental group of the manifold belongs one of the following cases:

(1) groups acting properly and isometrically on simply connected and non-positively curved manifolds [K], hyperbolic groups [CM],

(2) groups acting properly and isometrically on Hilbert spaces [HK],

(3) groups acting properly and isometrically on bolic spaces [KS],

(4) groups with finite asymptotic dimension [Y1],

(5) groups coarsely embeddable into Hilbert spaces [Y2] [H] [STY],

(6) groups coarsely embeddable into Banach spaces with property $(\mathrm{H})$ [KY],

(7) all linear groups and subgroups of all almost connected Lie groups [GHW],

(8) subgroups of the mapping class groups [Ha] [Ki],

(9) subgroups of $\operatorname{Out}\left(F_{n}\right)$, the outer automorphism groups of the free groups [BGH] ,

(10) groups acting properly and isometrically on (possibly infinite dimensional) admissible Hilbert-Hadamard spaces, in particular geometrically discrete subgroups of the group of volume preserving diffeomorphisms of any smooth compact manifold [GWY].

In the first three cases, an isometric action of a discrete group $\Gamma$ on a metric space $X$ is said to be proper if for some $x \in X, d(x, g x) \rightarrow \infty$ as $g \rightarrow \infty$, i.e. for any $x \in X$ and any positive number $R>0$, there exists a finite subset $F$ of $\Gamma$ such that $d(x, g x)>R$ if $g \in \Gamma-F$.

In a tour de force, Connes proved a striking theorem that the Novikov conjecture holds for higher signatures associated to Gelfand-Fuchs classes [C1. Connes, Gromov, and Moscovici proved the Novikov conjecture for higher signatures associated to Lipschitz group cohomology classes [CGM]. Hanke-Schick and Mathai proved the Novikov conjecture for higher signatures associated to group cohomology classes with degrees one and two [HS Ma.

J. Rosenberg discovered an important application of the (rational) strong Novikov conjecture to the existence problem of Riemannian metrics with positive scalar curvature $[\mathrm{R}]$. We refer to Rosenberg's survey [R1] for recent development on this topic. 
For the topological approach to the Novikov conjecture, we refer to the articles $[\mathrm{N}, \mathrm{FH}$, $\mathrm{CP}, \mathrm{DFW}, \mathrm{FW1}, \mathrm{W1}$.

Acknowledgment. The author wish to thank Xiaoman Chen, Paul, Baum, Alain Connes, Misha Gromov, Guihua Gong, Sherry Gong, Erik Guentner, Nigel Higson, Gennadi Kasparov, Vincent Lafforgue, Hervé Oyono-oyono, John Roe, Georges Skandalis, Xiang Tang, Romain Tessera, Jean-Louis Tu, Shmuel Weinberger, Rufus Willett, Jianchao Wu, Zhizhang Xie for inspiring discussions on the Novikov conjecture. The author would like to thank Sherry Gong, Hao Guo, and Slava Grigorchuk for very helpful comments on this article.

\section{Non-POSITIVELY CURVED GROUPS AND HYPERBOLIC GROUPS}

In this section, we give a survey on the work of A. Mishchenko, G. Kasparov, A. Connes and H. Moscovici, G. Kasparov and G. Skandalis on the Novikov conjecture for non-positively curved groups and hyperbolic groups.

In [M], A. Mishchenko introduced a theory of infinite dimensional Fredholm representations of discrete groups to prove the following theorem.

2.1. Theorem. The Novikov conjecture holds if the fundamental group of a manifold acts properly, isometrically and cocompactly on a simply connected manifold with non-positive sectional curvature.

In [K], G. Kasparov developed a bivariant K-theory, called KK-theory, to prove the following theorem.

2.2. Theorem. The Novikov conjecture holds if the fundamental group of a manifold acts properly and isometrically on a simply connected manifold with non-positive sectional curvature.

In the same article, G. Kasparov applied the above theorem to prove the following theorem.

2.3. Theorem. The Novikov conjecture holds if the fundamental group of a manifold is a discrete subgroup of a Lie group with finitely many connected components.

A. Connes and H. Moscovici proved the following theorem using powerful techniques from noncommutative geometry [CM].

2.4. Theorem. The Novikov conjecture holds if the fundamental group of a manifold is a hyperbolic group.

The theory of hyperbolic groups was developed by Gromov [G]. The proof of Theorem 2.5 uses Connes' theory of cyclic cohomology in a crucial way. Cyclic cohomology theory plays the role of de Rham theory in noncommutative geometry and is the natural receptacle for the Connes-Chern character [C].

The following theorem of G. Kasparov and G. Skandalis unified the above results [KS].

2.5. Theorem. The Novikov conjecture holds if the fundamental group of a manifold is bolic. 
Bolicity is a notion of non-positive curvature. Examples of bolic groups include hyperbolic groups and groups acting properly and isometrically on simply connected manifolds with non-positive sectional curvature.

\section{Amenable groups, groups With Finite asymptotic Dimension And COARSELY EMBEDDABLE GROUPS}

In this section, we give a survey on the work of Higson-Kasparov on the Novikov conjecture for amenable groups, my work on the Novikov conjecture for groups with finite asymptotic dimension, and the work of G. Yu, N. Higson, Skandalus-Tu-Yu on the Novikov conjecture for groups coarsely embeddable into Hilbert spaces. Finally we discuss the work of Kasparov-Yu on the connection of the Novikov conjecture with Banach space geometry.

Higson and Kasparov developed an index theory of certain differential operators on Hilbert space to prove the following theorem [HK].

3.1. Theorem. The Novikov conjecture holds if the fundamental group of a manifold acts properly and isometrically on a Hilbert space.

Recall that an isometric action $\alpha$ of a group $\Gamma$ on a Hilbert space is said to be proper if $\|\alpha(\gamma) h\| \rightarrow \infty$ when $\gamma \rightarrow \infty$, i.e. for any $h \in H$ and any positive number $R>0$, there exists a finite subset $F$ of $\Gamma$ such that $\|\alpha(\gamma) h\|>R$ if $\gamma \in \Gamma-F$. A theorem of BekkaCherix-Valette states that every amenable group acts properly and isometrically on Hilbert space [BCV]. Roughly speaking, a group is amenable if there exists large finite subsets of the group with small boundary. The concept of amenability is a large-scale geometric property and was introduced by von Neumann. We refer the readers to the book [NY] as a general reference for geometric group theory related to the Novikov conjecture.

Corollary. The Novikov conjecture holds if the fundamental group of a manifold is amenable.

This corollary is quite striking since the geometry of amenable groups can be very complicated (for example, the Grigorchuk groups [Gr]).

Next we recall a few basic concepts from geometric group theory. A non-negative function $l$ on a countable group $G$ is called a length function if $(1) l\left(g^{-1}\right)=l(g)$ for all $g \in G$; (2) $l(g h) \leq l(g)+l(h)$ for all $g$ and $h$ in $G$; (3) $l(g)=0$ if and only if $g=e$, the identity element of $G$. We can associate a left-invariant length metric $d_{l}$ to $l: d_{l}(g, h)=l\left(g^{-1} h\right)$ for all $g, h \in G$. A length metric is called proper if the length function is a proper map (i.e. the inverse image of every compact set is finite in this case). It is not difficult to show that every countable group $G$ has a proper length metric. If $l$ and $l^{\prime}$ are two proper length functions on $G$, then their associated length metrics are coarsely equivalent. If $G$ is a finitely generated group and $S$ is a finite symmetric generating set (in the sense that if an element is in $S$, then its inverse is also in $S$ ), then we can define the word length $l_{S}$ on $G$ by

$$
l_{S}(g)=\min \left\{n: g=s_{1} \cdots s_{n}, s_{i} \in S\right\} .
$$

If $S$ and $S^{\prime}$ are two finite symmetric generating sets of $G$, then their associated proper length metrics are quasi-isometric. 
The following concept is due to Gromov [G1].

3.1. Definition. The asymptotic dimension of a proper metric space $X$ is the smallest integer $n$ such that for every $r>0$, there exists a uniformly bounded cover $\left\{U_{i}\right\}$ for which the number of $U_{i}$ intersecting each $r$-ball $B(x, r)$ is at most $n+1$.

As example, the asymptotic dimension of $\mathbb{Z}^{n}$ is $n$, and the asymptotic dimension of the free group $\mathbb{F}_{n}$ with $n$ generators is 1 . Asymptotic dimension is invariant under coarse equivalence. The Lie group $G L(n, \mathbb{R})$ with a left-invariant Riemannian metric is quasi-isometric to $T(n, \mathbb{R})$, the subgroup of invertible upper triangular matrices. By permanence properties of asymptotic dimension [BD1], we know that the solvable group $T(n, \mathbb{R})$ has finite asymptotic dimension. As a consequence, every countable discrete subgroup of $G L(n, \mathbb{R})$ has finite asymptotic dimension (as a metric space with a proper length metric). More generally, one can prove that every discrete subgroup of an almost connected Lie group has finite asymptotic dimension (a Lie group is said to be almost connected if the number of its connected components is finite). Gromov's hyperbolic groups have finite asymptotic dimension [Roe2]. Mapping class groups also have finite asymptotic dimension [BBF].

In [Y1], I developed a quantitative operator K-theory to prove the following theorem.

3.2. Theorem. The Novikov conjecture holds if the fundamental group of a manifold has finite asymptotic dimension.

The following concept of Gromov makes precise the idea of drawing a good picture of a metric space in a Hilbert space.

3.2. Definition. (Gromov): Let $X$ be a metric space and $H$ be a Hilbert space. A map $f: X \rightarrow H$ is said to be a coarse embedding if there exist non-decreasing functions $\rho_{1}$ and $\rho_{2}$ on $[0, \infty)$ such that

(1) $\rho_{1}(d(x, y)) \leq d_{H}(f(x), f(y)) \leq \rho_{2}(d(x, y))$ for all $x, y \in X$;

(2) $\lim _{r \rightarrow+\infty} \rho_{1}(r)=+\infty$.

Coarse embeddability of a countable group is independent of the choice of proper length metrics. Examples of groups coarsely embeddable into Hilbert space include groups acting properly and isometrically on a Hilbert space (in particular amenable groups [BCV]), groups with Property A [Y2], countable subgroups of connected Lie groups [GHW], hyperbolic groups [S], groups with finite asymptotic dimension, Coxeter groups [DJ], mapping class groups [Ki, Ha], and semi-direct products of groups of the above types.

The following theorem unifies the above theorems.

3.3. Theorem. The Novikov conjecture holds if the fundamental group of a manifold is coarsely embeddable into Hilbert space.

Roughly speaking, this theorem says if we can draw a good picture of the fundamental group in a Hilbert space, then we can recognize the manifold at an infinitesimal level. This theorem was proved by myself when the classifying space of the fundamental group has the homotopy type of a finite CW complex Y2 and this finiteness condition was removed by 
N. Higson [H], Skandalis-Tu-Yu [STY]. The original proof of the above result makes heavy use of infinite diimensional analysis. More recently, R. Willett and myself found a relatively elementary proof within the framework of basic operator K-theory [WiY].

E. Guentner, N. Higson and S. Weinberger proved the beautiful theorem that linear groups are coarsely embeddable into Hilbert space [GHW]. Recall that a group is called linear if it is a subgroup of $G L(n, k)$ for some field $k$. The following theorem follows as a consequence GHW].

3.4. Theorem. The Novikov conjecture holds if the fundamental group of a manifold is a linear group.

More recently, Bestvina-Guirardel-Horbez proved that $\operatorname{Out}\left(F_{n}\right)$, the outer automorphism groups of the free group $F_{n}$, is coarsely embeddable into Hilbert space. This implies the following theorem $[\mathrm{BGH}]$.

3.5. Theorem. The Novikov conjecture holds if the fundamental group of a manifold is a subgroup of $\operatorname{Out}\left(F_{n}\right)$.

We have the following open question.

3.6. Open Question. Is every countable subgroup of the diffeomorphism group of the circle coarsely embeddable into Hilbert space?

Let $\mathfrak{E}$ be the smallest class of groups which include all groups coarsely embeddable into Hilbert space and is closed under direct limit. Recall that if $I$ is a directed set and $\left\{G_{i}, \phi_{i, j}\right\}_{i, j \in I, i<j}$ is a direct system of groups over $I$, then we can define the direct limit $\lim G_{i}$, where the homomorphism $\phi_{i j}: G_{i} \rightarrow G_{j}$ for $i<j$ is not necessary injective.

The following result is a consequence of Theorem 3.3.

3.7. Theorem. The Novikov conjecture holds if the fundamental group of a manifold is in the class $\mathfrak{E}$.

The following open question is a challenge to geometric group theorists.

3.8. Open Question. Is there any countable group not in the class $\mathfrak{E}$ ?

We mention that the Gromov monster groups are in class $\mathfrak{E}$ G2, G3, AD, O].

Next we shall discuss the connection of the Novikov conjecture with the geometry of Banach spaces.

3.3. Definition. A Banach space $X$ is said to have Property $(\mathrm{H})$ if there exist an increasing sequence of finite dimensional subspaces $\left\{V_{n}\right\}$ of $X$ and an increasing sequence of finite dimensional subspaces $\left\{W_{n}\right\}$ of a Hilbert space such that

(1) $V=\cup_{n} V_{n}$ is dense in $X$,

(2) if $W=\cup_{n} W_{n}, S(V)$ and $S(W)$ are respectively the unit spheres of $V$ and $W$, then there exists a uniformly continuous map $\psi: S(V) \rightarrow S(W)$ such that the restriction of $\psi$ to $S\left(V_{n}\right)$ is a homeomorphism (or more generally a degree one map) onto $S\left(W_{n}\right)$ for each $n$. 
As an example, let $X$ be the Banach space $l^{p}(\mathbb{N})$ for some $p \geq 1$. Let $V_{n}$ and $W_{n}$ be respectively the subspaces of $l^{p}(\mathbb{N})$ and $l^{2}(\mathbb{N})$ consisting of all sequences whose coordinates are zero after the $n$-th terms. We define a map $\psi$ from $S(V)$ to $S(W)$ by

$$
\psi\left(c_{1}, \cdots, c_{k}, \cdots\right)=\left(c_{1}\left|c_{1}\right|^{p / 2-1}, \cdots, c_{k}\left|c_{k}\right|^{p / 2-1}, \cdots\right) .
$$

$\psi$ is called the Mazur map. It is not difficult to verify that $\left\{V_{n}\right\},\left\{W_{n}\right\}$ and $\psi$ satisfy the conditions in the definition of Property $(\mathrm{H})$. For each $p \geq 1$, we can similarly prove that $C_{p}$, the Banach space of all Schatten $p$-class operators on a Hilbert space, has Property $(\mathrm{H})$.

Kasparov and Yu proved the following.

3.9. Theorem. The Novikov conjecture holds if the fundamental group of a manifold is coarsely embeddable into a Banach space with Property (H).

Let $c_{0}$ be the Banach space consisting of all sequences of real numbers converging to 0 with the sup norm.

3.10. Open Question. Does the Banach space $c_{0}$ have Property $(\mathrm{H})$ ?

A positive answer to this question would imply the Novikov conjecture since every countable group admits a coarse embedding into $c_{0}[\mathrm{BG}]$.

A less ambitious question is the following.

3.11. Open Question. Is every countable subgroup of the diffeomorphism group of a compact smooth manifold coarsely embeddable into $C_{p}$ for some $p \geq 1$ ?

For each $p>q \geq 2$, it is also an open question to construct a bounded geometry space which is coarsely embeddable into $l^{p}(\mathbb{N})$ but not $l^{q}(\mathbb{N})$. Beautiful results in [JR] and [MN] indicate that such a construction should be possible. Once such a metric space is constructed, the next natural question is to construct countable groups which coarsely contain such a metric space. These groups would be from another universe and would be different from any group we currently know.

\section{Gelfand-Fuchs Classes, the Group of volume PRESERVing Diffeomorphisms, HiLBERT-HADAMARD SPACES}

In this section, we give an overview on the work of A. Connes, Connes-Gromov-Moscovici on the Novikov conjecture for Gelfand-Fuchs classes and the recent work of Gong-Wu-Yu on the Novikov conjecture for groups acting properly and isometrically on Hilbert-Hadamard spaces and for any geometrically discrete subgroup of the group of volume preserving diffeomorphisms of a compact smooth manifold.

A. Connes proved the following deep theorem on the Novikov conjecture [C1].

4.1. Theorem. The Novikov conjecture holds for higher signatures associated to the GelfandFuchs cohomology classes of a subgroup of the group of diffeomorphisms of a compact smooth manifold.

The proof of this theorem uses the full power of noncommutative geometry [C]. 
4.2. Open Question. Does the Novikov conjecture hold for any subgroup of the group of diffeomorphisms of a compact smooth manifold?

Motivated in part by this open question, S. Gong, J. Wu and G. Yu prove the following theorem [GWY].

4.3. Theorem. The Novikov conjecture holds for groups acting properly and isometrically on an admissible Hilbert-Hadamard space.

Roughly speaking, Hilbert-Hadamard spaces are (possibly infinite dimensional) simply connected spaces with non-positive curvature. We will give a precise definition a little later. We say that a Hilbert-Hadamard space $M$ is admissible if it has a sequence of subspaces $M_{n}$, whose union is dense in $M$, such that each $M_{n}$, seen with its inherited metric from $M$, is isometric to a finite-dimensional Riemannian manifold. Examples of admissible HilbertHadamard spaces include all simply connected and non-positively curved Riemannian manifold, the Hilbert space, and certain infinite dimensional symmetric spaces. Theorem 4.3 can be viewed as a generalization of both Theorem 2.1 and Theorem 3.1.

Infinite dimensional symmetric spaces are often naturally admissible Hilbert-Hadamard spaces. One such an example of an admissible infinite-dimensional symmetric space is

$$
L^{2}(N, \omega, \operatorname{SL}(n, \mathbb{R}) / \mathrm{SO}(n)),
$$

where $N$ is a compact smooth manifold with a given volume form $\omega$. This infinite-dimensional symmetric space is defined to be the completion of the space of all smooth maps from $N$ to $X=\mathrm{SL}(n, \mathbb{R}) / \mathrm{SO}(n)$ with respect to the following distance:

$$
d(\xi, \eta)=\left(\int_{N}\left(d_{X}(\xi(y), \eta(y))\right)^{2} d \omega(y)\right)^{\frac{1}{2}},
$$

where $d_{X}$ is the standard Riemannian metric on the symmetric space $X$ and $\xi$ and $\eta$ are two smooth maps from $N$ to $X$. This space can be considered as the space of $L^{2}$-metrics on $N$ with the given volume form $\omega$ and is Hilbert-Hadamard space. With the help of this infinite dimensional symmetric space, the above theorem can be applied to study the Novikov conjecture for geometrically discrete subgroups of the group of volume preserving diffeomorphisms on such a manifold.

The key ingredients of the proof for Theorem 4.3 include a construction of a $C^{*}$-algebra modeled after the Hilbert-Hadamard space, a deformation technique for the isometry group of the Hilbert-Hadamard space and its corresponding actions on $K$-theory, and a $K K$-theory with real coefficient developed by Antonini, Azzali, and Skandalis [AAS].

Let $\operatorname{Diff}(N, \omega)$ denote the group of volume preserving diffeomorphisms on a compact orientable smooth manifold $N$ with a given volume form $\omega$. In order to define the concept of geometrically discrete subgroups of $\operatorname{Diff}(N, \omega)$, let us fix a Riemannian metric on $N$ with the given volume $\omega$ and define a length function $\lambda$ on $\operatorname{Diff}(N, \omega)$ by:

$$
\lambda_{+}(\varphi)=\left(\int_{N}(\log (\|D \varphi\|))^{2} d \omega\right)^{1 / 2}
$$


and

$$
\lambda(\varphi)=\max \left\{\lambda_{+}(\varphi), \lambda_{+}\left(\varphi^{-1}\right)\right\}
$$

for all $\varphi \in \operatorname{Diff}(N, \omega)$, where $D \varphi$ is the Jacobian of $\varphi$, and the norm $\|\cdot\|$ denotes the operator norm, computed using the chosen Riemannian metric on $N$.

4.1. Definition. A subgroup $\Gamma$ of $\operatorname{Diff}(N, \omega)$ is said to be a geometrically discrete subgroup if $\lambda(\gamma) \rightarrow \infty$ when $\gamma \rightarrow \infty$ in a $\Gamma$, i.e. for any $R>0$, there exists a finite subset $F \subset \Gamma$ such that $\lambda(\gamma) \geq R$ if $\gamma \in \Gamma \backslash F$.

Observe that although the length function $\lambda$ depends on our choice of the Riemannian metric, the above notion does not. Also notice that if $\gamma$ preserves the Riemannian metric we chose, then $\lambda(g)=0$. This suggests that the class of geometrically discrete subgroups of $\operatorname{Diff}(N, \omega)$ doesn't intersect with the class of groups of isometries. Of course we already know the Novikov conjecture for any group of isometries on a compact Riemannian manifold. This, together with the following result, gives an optimistic perspective on the open question on the Novikov conjecture for groups of volume preserving diffeomorphisms.

4.4. Theorem. Let $N$ be a compact smooth manifold with a given volume form $\omega$, and let $\operatorname{Diff}(N, \omega)$ be the group of all volume preserving diffeomorphisms of $N$. The Novikov conjecture holds for any geometrically discrete subgroup of $\operatorname{Diff}(N, \omega)$.

Next we will give a precise definition of Hilbert-Hadamard space.

We will first recall the concept of $\mathrm{CAT}(0)$ spaces. Let $X$ be a geodesic metric space. Let $\Delta$ be a triangle in $X$ with geodesic segments as its sides. $\Delta$ is said to satisfy the $\operatorname{CAT}(0)$ inequality if there is a comparison triangle $\Delta^{\prime}$ in Euclidean space, with sides of the same length as the sides of $\Delta$, such that distances between points on $\Delta$ are less than or equal to the distances between corresponding points on $\Delta^{\prime}$. The geodesic metric space $X$ is said to be a $\mathrm{CAT}(0)$ space if every geodesic triangle satisfies the $\mathrm{CAT}(0)$ inequality.

Let $X$ be a geodesic metric space. For three distinct points $x, y, z \in X$, we define the comparison angle $\widetilde{L} x y z$ to be

$$
\widetilde{\angle} x y z=\arccos \left(\frac{d(x, y)^{2}+d(y, z)^{2}-d(x, z)^{2}}{2 d(x, y) d(y, z)}\right) .
$$

In other words, $\widetilde{\angle} x y z$ can be thought of as the angle at $y$ of a triangle $A B C$ in the Euclidean plane with side-lengths that agree with the side-lengths of the geodesic triangle $x y z$ in $X$.

Given two nontrivial geodesic paths $\alpha$ and $\beta$ emanating from a point $p$ in $X$, meaning that $\alpha(0)=\beta(0)=p$, we define the angle between them, $\angle(\alpha, \beta)$, to be

$$
\angle(\alpha, \beta)=\lim _{s, t \rightarrow 0} \tilde{Z}(\alpha(s), p, \beta(t)),
$$

provided that the limit exists. For CAT(0) spaces, since the comparison angle $\widetilde{Z}(\alpha(s), p, \beta(t))$ decreases with $s$ and $t$, the angle between any two geodesic paths emanating from a point is well-defined. The above angles satisfy the triangle inequality. 
For a point $p \in X$, let $\Sigma_{p}^{\prime}$ denote the metric space induced from the space of all geodesics emanating from $p$ equipped with the pseudometric of angles, that is, for geodesics $\alpha$ and $\beta$, we define $d(\alpha, \beta)=\angle(\alpha, \beta)$. Note, in particular, from our definition of angles, that $d(\alpha, \beta) \leq \pi$ for any geodesics $\alpha$ and $\beta$.

We define $\Sigma_{p}$ to be the completion of $\Sigma_{p}^{\prime}$ with respect to the distance $d$. The tangent cone $K_{p}$ at a point $p$ in $X$ is then defined to be a metric space which is, as a topological space, the cone of $\Sigma_{p}$. That is, topologically

$$
K_{p} \simeq \Sigma_{p} \times[0, \infty) / \Sigma_{p} \times\{0\} .
$$

The metric on it is given as follows. Any two points $p, q \in K_{p}$ can expressed as $p=[(x, t)]$ and $q=[(y, s)]$. The metric is given by

$$
d(p, q)=\sqrt{t^{2}+s^{2}-2 s t \cos (d(x, y))} .
$$

The distance is what the distance would be if we went along geodesics in a Euclidean plane with the same angle between them as the angle between the corresponding directions in $X$.

The following definition is inspired by [FS].

4.5. Definition. A Hilbert-Hadamard space is a complete geodesic CAT(0) metric space (i.e., an Hadamard space) all of whose tangent cones are isometrically embedded in Hilbert spaces.

Every connected and simply connected Riemannian-Hilbertian manifold with non-positive sectional curvature is a separable Hilbert-Hadamard space. In fact, a Riemannian manifold without boundary is a Hilbert-Hadamard space if and only if it is complete, connected, and simply connected, and has nonpositive curvature. We remark that a $\operatorname{CAT}(0)$ space $X$ is always uniquely geodesic.

Recall that a subset of a geodesic metric space is called convex if it is again a geodesic metric space when equipped with the restricted metric. We observe that a closed convex subset of a Hilbert-Hadamard space is itself a Hilbert-Hadamard space.

4.2. Definition. A separable Hilbert-Hadamard space $M$ is called admissible if there is a sequence of convex subsets isometric to finite-dimensional Riemannian manifolds whose union is dense in $M$.

The notion of Hilbert-Hadamard spaces is more general than simply connected RiemannianHilbertian space with non-positive sectional curvature. For example, the infinite dimensional symmetric space $L^{2}(N, \omega, \mathrm{SL}(n, \mathbb{R}) / \mathrm{SO}(n))$ is a Hilbert-Hadamard space but not a Riemannian-Hilbertian space with non-positive sectional curvature.

\section{Geometric COMPlexity, topological Rigidity AND NON-RIGIDITy}

An integral version of the Novikov conjecture implies the stable Borel conjecture [J, FH] which states that all compact aspherical manifolds are stably topologically rigid, i.e. if another compact manifold $N$ is homotopy equivalent to the given compact aspherical manifold $M$, then $N \times \mathbb{R}^{n}$ is homeomorphic to $M \times \mathbb{R}^{n}$ for some $n$. In this section, we discuss the 
concept of decomposition complexity introduced by Guentner-Tessera-Yu [GTY] and its applications to the stable Borel conjecture. The method used here is very much inspired by the quantitative operator K-theory approach developed in [Y1]. We shall also briefly discuss the application of the Novikov conjecture to non-rigidity of manifolds in Weinberger-Xie-Yu WXY.

We shall first recall the concept of finite decomposition complexity.

For any $r>0$, a collection of subspaces $\left\{Z_{i}\right\}$ of a metric space $Z$ is said to be $r$-disjoint if for all $i \neq j$ we have $d\left(Z_{i}, Z_{j}\right) \geq r$. To express the idea that $Z$ is the union of subspaces $Z_{i}$, and that the collection of these subspaces is $r$-disjoint we write

$$
Z=\bigsqcup_{r-\text { disjoint }} Z_{i}
$$

A family of metric spaces $\left\{Z_{i}\right\}$ is called bounded if there is a uniform bound on the diameter of the individual $Z_{i}$ :

$$
\sup \operatorname{diameter}\left(Z_{i}\right)<\infty \text {. }
$$

5.1. Definition. A family of metric spaces $\{X\}$ is $r$-decomposable over another family of metric spaces $\{Y\}$ if every $X \in\{X\}$ admits an $r$-decomposition

$$
X=X_{0} \cup X_{1}, \quad X_{i}=\bigsqcup_{r-\text { disjoint }} X_{i j},
$$

where each $X_{i j} \in\{Y\}$.

5.2. Definition. Let $\Omega$ be a collection of families of metric spaces. A family of metric spaces $\{X\}$ is said to be decomposable over $\Omega$ if, for every $r>0$, there exists a family of metric spaces $\{Y\} \in \Omega$ and an $r$-decomposition of $\{X\}$ over $\{Y\}$. The collection $\Omega$ is said to be stable under decomposition if every family of metric spaces which decomposes over $\Omega$ actually belongs to $\Omega$.

5.3. Definition. The collection $\mathfrak{D}$ of families of metric spaces with finite decomposition complexity is the minimal collection of families of metric spaces containing all bounded families of metric spaces and stable under decomposition. We abbreviate membership in $\mathfrak{D}$ by saying that a family of metric spaces in $\mathfrak{D}$ has finite decomposition complexity. A metric space $X$ is said to have finite decomposition complexity if the family consisting of only $X$ has finite decomposition complexity.

Observe that finite decomposition complexity is invariant under coarse equivalence. We also remark that metric spaces with finite decomposition complexity are coarsely embeddable into Hilbert space.

By the definition of asymptotic dimension, any proper metric space with asymptotic dimension at most 1 has finite decomposition complexity. More generally, a metric space with finite asymptotic dimension has finite decomposition complexity. This fact follows from a 
theorem of Dranishnikov and Zarichnyi stating that a proper metric space with finite asymptotic dimension is coarsely equivalent to a subspace of the product of finitely many trees.

Let $G=\oplus_{k=1}^{\infty} \mathbb{Z}$ be the countable group with the proper length metric associated to the length function $l$ :

$$
l\left(\oplus_{k=1}^{\infty} n_{k}\right)=\sum_{k=1}^{\infty} k\left|n_{k}\right|
$$

for each $\oplus_{k=1}^{\infty} n_{k} \in G$. For each $r>0$, let $k_{0}$ be the smallest integer greater than $r$. For each $\alpha \in \oplus_{k=k_{0}}^{\infty} \mathbb{Z}$, let

$$
G_{\alpha}=\left\{\oplus_{k=1}^{k_{0}-1} n_{k} \oplus \alpha: \oplus_{k=1}^{k_{0}-1} n_{k} \in \oplus_{k=1}^{k_{0}-1} \mathbb{Z}\right\} .
$$

Notice that $G=\bigsqcup_{r \text {-disjoint }} G_{\alpha}$ and that $G_{\alpha}$ is (uniformly) coarsely equivalent to $\oplus_{k=1}^{k_{0}-1} \mathbb{Z}$. This implies that $\left\{G_{\alpha}\right\} \in \mathfrak{D}$. It follows that $G$ has finite decomposition complexity despite the fact that $G$ has infinite asymptotic dimension. If $H \subset G L(2, \mathbb{R})$ is the finitely generated group consisting of all matrices of the form $\left(\begin{array}{cc}\pi^{n} & p(\pi) \\ 0 & \pi^{-n}\end{array}\right)$, with a little extra work we can show that $H$ has finite decomposition complexity.

More generally, we have the following result from [GTY].

5.1. Theorem. Any countable subgroup of $G L(n, k)$ has finite decomposition complexity (as a metric space with a proper length metric), where $k$ is a field.

The same result is true for any countable subgroup of an almost connected Lie group and any countable elementary amenable group [GTY].

The following result is proved in [GTY].

5.2. Theorem. The stable Borel conjecture holds for aspherical manifolds whose fundamental groups have finite decomposition complexity.

We have the following open question.

5.3. Open Question. Does any countable amenable group have finite decomposition complexity?

In particular, it remains an open question whether the Grigorchuk groups have finite decomposition complexity.

Finally, we ask the following open question.

5.4. Open Question. Does the group $\operatorname{Out}\left(F_{n}\right)$ have finite decomposition complexity?

There has been spectacular recent progress on the Borel conjecture. We will not attempt to survey all important results, but only mention the fundamental work of Farrell-Jones [FJ1, FJ2, FJ3, FJ4], Bartels-Lück [BL], and Bartels-Lück-Holger [BLH]. I also refer interested readers to the beautiful books by T. Farrell and L. Jones [FJ2] and S. Weinbeger [W]. 
Finally we mention interesting applications of the Novikov conjecture to non-rigidity of manifolds [WY, WXY]. In particular, Weinberger-Xie-Yu introduced the Novikov rho invariant to prove that the structure groups of certain manifolds are infinitely generated [WXY]. Recall that the topological structure group $S^{T O P}(M)$ is the abelian group of equivalence classes of all pairs $(f, X)$ such that $X$ is a closed oriented manifold and $f: X \rightarrow M$ is an orientation-preserving homotopy equivalence. The structure group $S^{T O P}(M)$ measures non-rigidity of $M$.

5.5. Theorem. Let $M$ be a closed oriented topological manifold of dimension $n \geq 5$, and $\Gamma$ be its fundamental group. Suppose the rational Kasparov-Baum-Connes assembly map for $\Gamma$ is split injective. If $\oplus_{k \in \mathbb{Z}} H_{n+1+4 k}(\Gamma, \mathbb{C})$ is infinitely generated, then the topological structure group of $S^{T O P}(M)$ is infinitely generated.

We refer to the article [WXY] for examples of groups satisfying conditions in the above theorem. We remark that the condition on split injectivity of the rational Kasparov-BaumConnes assembly map is one version of the strong Novikov conjecture.

\section{REFERENCES}

[AAS] P. Antonini, S. Azzali, G. Skandalis. The Baum-Connes conjecture localised at the unit element of a discrete group arXiv:1807.05892, 2018.

[AD] G.N. Arzhantseva, T. Delzant. Examples of random groups Preprint, 2008.

[B] A. BARTELS. Squeezing and higher algebraic K-theory. K-Theory 28 (2003), no. 1, 19-37.

[BC] P. BAum, A. Connes. K-theory for discrete groups Operator algebras and applications, Vol. 1, volume 135 of London Mathematical Society, pages 1-20. Cambridge Univ. Press, Cambridge, 1988.

[BCH] P. Baum, A. Connes, N. Higson. Classifying space for proper actions and K-theory of group C*-algebras. C*-algebras: 1943-1993 (San Antonio, TX, 1993), 240-291, Contemp. Math., 167, Amer. Math. Soc., Providence, RI, 1994.

[BL] A. BARTELs, W. LüCK. The Borel Conjecture for hyperbolic and CAT(0)-groups. Ann. of Math. (2) 175 (2012), no. 2, 631-689.

[BLH] A. BARTELS, W. LüCK, R. HOLGER. The K-theoretic Farrell-Jones conjecture for hyperbolic groups. Invent. Math. 172 (2008), no. 1, 29-70.

[BR] A. Bartels, D. Rosenthal. On the $K$-theory of groups with finite asymptotic dimension. J. Reine Angew. Math. 612 (2007), 35-57.

[BD1] G. Bell, A. Dranishnikov. On asymptotic dimension of groups. Algebr. Geom. Topol. 1 (2001), 57-71 (electronic).

[BD2] G. Bell, A. Dranishnikov. A Hurewicz-type theorem for asymptotic dimension and applications to geometric group theory. Transactions American Math. Soc. Volume 358, Number 11, 2006, 4749-4764.

[BCV] M. Bekka, P. Cherix, A. Valette. Proper affine isometric actions of amenable groups. Novikov conjectures, index theorems and rigidity, Vol. 2 (Oberwolfach, 1993), 1-4, London Math. Soc. Lecture Note Ser., 227, Cambridge Univ. Press, Cambridge, 1995.

[BBF] M. Bestvina, K. Bromberg, K. Fujiwara. Constructing group actions on quasi-trees and applications to mapping class groups. Publ. Math. Inst. Hautes Études Sci. 122 (2015), 1-64.

[BGH] M. Bestvina, V. Guirardel, C. Horbez. Boundary amenability of Out $\left(F_{N}\right)$. arXiv:1705.07017, 2017. 
[BG] N. Brown, E. Guentner. Uniform embeddings of bounded geometry spaces into reflexive Banach space. Proc. Amer. Math. Soc. 133 (2005), no. 7, 2045-2050.

[CG] G. Carlsson, B. Goldfarb. The integral K-theoretic Novikov conjecture for groups with finite asymptotic dimension. Invent. Math. 157 (2004), no. 2, 405-418.

[CP] G. Carlsson, E. Pedersen. Controlled algebra and the Novikov conjectures for $K$ - and L-theory. Topology 34 (1995), no. 3, 731-758.

[CFY] S. Chang, S. Ferry, G. Yu. Bounded rigidity of manifolds and asymptotic dimension growth. J. K-Theory 1 (2008), no. 1, 129-144.

[C] A. Connes. Noncommutative Geometry. Academic Press, 1994.

[C1] A. Connes. Cyclic cohomology and the transverse fundamental class of a foliation. Geometric methods in operator algebras (Kyoto, 1983), pages 52-144, volume 123, Pitman Res. Notes Math. Ser., 1986.

[CH] A. Connes, N. Higson. Déformations, morphismes asymptotiques et K-théorie bivariante. (French) [Deformations, asymptotic morphisms and bivariant K-theory] C. R. Acad. Sci. Paris Sér. I Math. 311 (1990), no. 2, 101-106.

[CM] A. Connes, H. Moscovici. Cyclic cohomology, the Novikov conjecture and hyperbolic groups. Topology 29 (1990), no. 3, 345-388.

[CGM] A. Connes, M. Gromov, H. Moscovici. Group cohomology with Lipschitz control and higher signatures. Geom. Funct. Anal. 3 (1993), no. 1, 1-78.

[D] M. DAvIs. Groups generated by reflections and aspherical manifolds not covered by Euclidean space. Ann. of Math. (2) 117 (1983), no. 2, 293-324.

[DFW] A. Dranishnikov, S. Ferry, S. WeinBerger. An etale approach to the Novikov conjecture. Comm. Pure Appl. Math. 61 (2008), no. 2, 139-155.

[DJ] A. Dranishnikov, T. Januszkiewicz. Every Coxeter group acts amenably on a compact space. Proceedings of the 1999 Topology and Dynamics Conference (Salt Lake City, UT). Topology Proc. 24 (1999), Spring, 135-141.

[FH] F. T. FARRELL, W. C. Hsiang. On Novikov's conjecture for nonpositively curved manifolds. Ann. of Math. (2) 113 (1981), no. 1, 199-209.

[FJ1] T. FArrell, L. Jones. A topological analogue of Mostow's rigidity theorem. J. Amer. Math. Soc. 2 (1989), no. 2, 257-370.

[FJ2] T. FARrell, L. Jones. Classical aspherical manifolds. CBMS Regional Conference Series in Mathematics, 75. Published for the Conference Board of the Mathematical Sciences, Washington, DC; by the American Mathematical Society, Providence, RI, 1990.

[FJ3] T. FARrell, L. Jones. Topological rigidity for compact non-positively curved manifolds. Differential geometry: Riemannian geometry (Los Angeles, CA, 1990), 229-274, Proc. Sympos. Pure Math., 54, Part 3, Amer. Math. Soc., Providence, RI, 1993.

[FJ4] T. FArrell, L. Jones. Rigidity for aspherical manifolds with $\pi_{1} \subset G L_{m}(R)$. Asian J. Math. 2 (1998), no. 2, 215-262.

[FP] S. FERry, E. Pedersen. Epsilon surgery theory. Novikov conjectures, index theorems and rigidity, Vol. 2 (Oberwolfach, 1993), 167-226, London Math. Soc. Lecture Note Ser., 227, Cambridge Univ. Press, Cambridge, 1995.

[FS] D. Fisher, L. Silberman. Groups not acting on manifolds. Int. Math. Res. Not. IMRN 2008, no. 16, Art. ID rnn060, $11 \mathrm{pp.}$

[FW1] S. Ferry, S. WeinBerger. Curvature, tangentiality, and controlled topology. Invent. Math. 105 (1991), no. 2, 401-414.

[FW2] S. FERry, S. Weinberger. A coarse approach to the Novikov conjecture. Novikov conjectures, index theorems and rigidity, Vol. 1 (Oberwolfach, 1993), 147-163, London Math. Soc. Lecture Note Ser., 226, Cambridge Univ. Press, Cambridge, 1995. 
[GWY] S. Gong, J. Wu, G. YU. The Novikov conjecture, the group of volume preserving diffeomorphisms and Hilbert-Hadamard spaces. arXiv:1811.02086, 2018.

[G] M. Gromov. Hyperbolic groups. In Gersten, Steve M. Essays in group theory. Mathematical Sciences Research Institute Publications. 8. New York: Springer. pp. 75-263.

[G1] M. Gromov. Asymptotic invariants of infinite groups. Geometric group theory, Vol. 2 (Sussex, 1991), 1-295, London Math. Soc. Lecture Note Ser., 182, Cambridge Univ. Press, Cambridge, 1993.

[G2] M. Gromov. Spaces and questions. GAFA 2000 (Tel Aviv, 1999). Geom. Funct. Anal. 2000, Special Volume, Part I, 118-161.

[G3] M. Gromov. Random walks in random groups. Geom. Funct. Anal. 13 (2003), no. 1, 73-146.

[Gr] R. I. Grigorchuk. Degrees of growth of finitely generated groups and the theory of invariant means. (Russian) Izv. Akad. Nauk SSSR Ser. Mat. 48 (1984), no. 5, 939-985.

[GHW] E. Guentner, N. Higson, S. Weinberger. The Novikov Conjecture for Linear Groups. Publ. Math. Inst. Hautes Études Sci. No. 101 (2005), 243-268.

[GTY] E. Guentner, R. Tessera, G. Yu. A notion of geometric complexity and its application to topological rigidity. Invent. Math. 189 (2012), no. 2, 315-357.

[GWY] E. Guentner, R. Willett, G. Yu. Dynamical complexity and controlled operator K-theory. Groups, Geometry and Dynamics, Vol. 7, 2 (2013) 377-402.

[Ha] U. HamenstäDt. Geometry of the mapping class groups. I. Boundary amenability. Invent. Math. 175 (2009), no. 3, 545-609.

[H] N. Higson. Bivariant K-theory and the Novikov conjecture. Geom. Funct. Anal. 10 (2000), no. 3, $563-581$.

[HK] N. Higson, G. Kasparov. E -theory and KK-theory for groups which act properly and isometrically on Hilbert space. Invent. Math. 144 (2001), no. 1, 23-74.

[HS] S. Hanke, T. Schick. The strong Novikov conjecture for low degree cohomology. Geom. Dedicata 135 (2008), 119-127.

[HiS] M. Hilsum, G. Skandalis. Georges Invariance par homotopie de la signature à coefficients dans un fibré presque plat. (French) [Homotopy invariance of the signature with coefficients in an almost flat fiber bundle]. J. Reine Angew. Math. 423 (1992), 73-99.

[J] L. J. The integral Novikov conjectures for linear groups containing torsion elements. J. Topol. 1 (2008), no. 2, 306-316.

[JR] W. B. Johnson, N. L. Randrianarivony. $l_{p}(p>2)$ does not coarsely embed into a Hilbert space. Proc. Amer. Math. Soc. 134 (2006), 1045-1050 (electronic).

[K] G. KASPAROv. Equivariant KK-theory and the Novikov conjecture. Invent. Math. 91 (1988), no. $1,147-201$.

[KS] G. Kasparov, G. Skandalis. Groups acting properly on "bolic" spaces and the Novikov conjecture. Ann. of Math. (2) 158 (2003), no. 1, 165-206.

[KY] G. Kasparov, G. Yu. The Novikov conjecture and geometry of Banach spaces. Geometry and Topology 16 (2012), no. 3, 1859-1880.

[Ki] Y. KIDA. The mapping class group from the viewpoint of measure equivalence theory. Mem. Amer. Math. Soc. 196 (2008), no. 916.

[Ma] V. Mathai. The Novikov conjecture for low degree cohomology classes. Geom. Dedicata 99 (2003), $1-15$.

[M] A. S. MishCHENKo. Infinite-dimensional representations of discrete groups, and higher signatures. (Russian) Izv. Akad. Nauk SSSR Ser. Mat. 38 (1974), 81-106.

[MN] M. Manor, A. Naor. Metric cotype. Ann. of Math. (2) 168 (2008), no. 1, 247-298.

$[\mathrm{N}] \quad$ S. P. Novikov. Algebraic construction and properties of Hermitian analogs of $k$-theory over rings with involution from the point of view of Hamiltonian formalism. Some applications to differential 
topology and to the theory of characteristic classes. Izv. Akad. Nauk SSSR, v. 34, 1970 I N2, pp. 253-288; II: N3, pp. 475-500.

[N1] S. P. Novikov. Topological invariance of rational classes of Pontrjagin. (Russian) Dokl. Akad. Nauk SSSR 1631965 298-300.

[NY] P. Nowak, G. YU. Large scale geometry. European Mathematical Society Publishing House, 2012.

[O] D. OsAJDA. Small cancellation labellings of some infinite graphs and applications. arXiv:1406.5015, 2014.

[OY] H. Oyono-Oyono, G. Yu. On quantitative operator K-theory. Ann. Inst. Fourier (Grenoble) 65 (2015), no. 2, 605-674.

[RTY] D. Ramras, R. Tessera, G. Yu. Finite decomposition complexity and the integral Novikov conjecture for higher algebraic K-theory. J. Reine Angew. Math. 694 (2014), 129-178.

[Roe] J. Roe. Coarse cohomology and index theory on complete Riemannian manifolds. Mem. Amer. Math. Soc. 104 (1993), no. 497.

[Roe1] J. RoE. Index theory, coarse geometry, and topology of manifolds. CBMS Regional Conference Series in Mathematics, 90. Published for the Conference Board of the Mathematical Sciences, Washington, DC; by the American Mathematical Society, Providence, RI, 1996.

[Roe2] J. RoE, Hyperbolic groups have finite asymptotic dimension. Proc. Amer. Math. Soc. 133 (2005), no. 9, 2489-2490.

[R] J. Rosenberg. $C^{*}$-algebras, positive scalar curvature, and the Novikov conjecture. Inst. Hautes Études Sci. Publ. Math. No. 58 (1983), 197-212 (1984).

[R1] J. Rosenberg. Manifolds of positive scalar curvature: a progress report. Surveys in differential geometry. Vol. XI, 259-294, Surv. Differ. Geom., 11, Int. Press, Somerville, MA, 2007.

[S] Z. SELA. Uniform embeddings of hyperbolic groups in Hilbert spaces. Israel J. Math. 80 (1992), no. 1-2, 171-181.

[STY] G. Skandalis, J. Tu, G. Yu. The coarse Baum-Connes conjecture and groupoids. Topology 41 (2002), no. 4, 807-834.

[W] S. WeinBerger. Variations on a theme of Borel. Book draft, available at http://math.uchicago.edu/ shmuel/VTBdraft.pdf

[W1] S. WeinBerger. Aspects of the Novikov conjecture. Geometric and topological invariants of elliptic operators (Brunswick, ME, 1988), 281-297, Contemp. Math., 105, Amer. Math. Soc., Providence, RI, 1990.

[WXY] S. Weinberger, Z. XIE, G. YU. Additivity of higher rho invariants and nonrigidity of topological manifolds. to appear in Communications in Pure and Applied Mathematics.

[WY] S. WeinBerger, G. YU. Finite part of operator K-theory for groups finitely embeddable into Hilbert space and the degree of nonrigidity of manifolds. Geom. Topol. 19 (2015), no. 5, 2767-2799.

[WiY] R. Willett, G. Yu. Higher index theory. Book draft, available at https://math.hawaii.edu/ rufus/Skeleton.pdf, 2018.

[Y1] G. YU. The Novikov conjecture for groups with finite asymptotic dimension. Ann. of Math. (2) 147 (1998), no. 2, 325-355.

[Y2] G. YU. The coarse Baum-Connes conjecture for spaces which admit a uniform embedding into Hilbert space. Invent. Math. 139 (2000), no. 1, 201-240.

Department of Mathematics, Mailstop 3368, Texas A\&M University, College Station, TX 77843, USA

E-mail address: guoliangyu@math.tamu.edu 